\title{
Chromatin environment, transcriptional regulation, and splicing distinguish lincRNAs and mRNAs
}

\author{
Marta Melé, 1,2 Kaia Mattioli, 1,2,3 William Mallard, ${ }^{1,2}$ David M. Shechner, ${ }^{1,2}$ \\ Chiara Gerhardinger, ${ }^{1,2}$ and John L. Rinn ${ }^{1,2,4}$ \\ ${ }^{1}$ Department of Stem Cell and Regenerative Biology, Harvard University, Cambridge, Massachusetts 02138, USA; ${ }^{2}$ Broad Institute \\ of Massachusetts Institute of Technology and Harvard, Cambridge, Massachusetts 02142, USA; ${ }^{3}$ Department of Biological \\ and Biomedical Sciences, Harvard University, Boston, Massachusetts 02115, USA; ${ }^{4}$ Department of Pathology, Beth Israel Deaconess \\ Medical Center, Boston, Massachusetts 02215, USA
}

\begin{abstract}
While long intergenic noncoding RNAs (lincRNAs) and mRNAs share similar biogenesis pathways, these transcript classes differ in many regards. LincRNAs are less evolutionarily conserved, less abundant, and more tissue-specific, suggesting that their pre- and post-transcriptional regulation is different from that of mRNAs. Here, we perform an in-depth characterization of the features that contribute to lincRNA regulation in multiple human cell lines. We find that lincRNA promoters are depleted of transcription factor (TF) binding sites, yet enriched for some specific factors such as GATA and FOS relative to mRNA promoters. Surprisingly, we find that H3K9me3-a histone modification typically associated with transcriptional repression-is more enriched at the promoters of active lincRNA loci than at those of active mRNAs. Moreover, H3K9me3-marked lincRNA genes are more tissue-specific. The most discriminant differences between lincRNAs and mRNAs involve splicing. LincRNAs are less efficiently spliced, which cannot be explained by differences in U1 binding or the density of exonic splicing enhancers but may be partially attributed to lower U2AF65 binding and weaker splicing-related motifs. Conversely, the stability of lincRNAs and mRNAs is similar, differing only with regard to the location of stabilizing protein binding sites. Finally, we find that certain transcriptional properties are correlated with higher evolutionary conservation in both DNA and RNA motifs and are enriched in lincRNAs that have been functionally characterized.
\end{abstract}

[Supplemental material is available for this article.]

Transcription and post-transcriptional regulation are crucial processes for the biogenesis and function of all RNA species. While several noncoding RNA classes-such as microRNAs, snoRNAs, and tRNAs—-have post-transcriptional processing pathways that are unique to each class, long intergenic noncoding RNA (lincRNA) biogenesis shares many similarities with that of protein-coding mRNAs (Quinn and Chang 2015). LincRNAs are transcribed by RNA Polymerase II, 5' -capped, spliced, and polyadenylated. Like those of mRNAs, lincRNA splice site dinucleotides are canonical, suggesting that they use the same splicing regulatory signals as mRNAs (Derrien et al. 2012). However, lincRNAs differ from mRNAs in several regards: They have fewer (although usually longer) exons (Derrien et al. 2012), they exhibit more tissue-specific expression (Cabili et al. 2011; Ulitsky and Bartel 2013), and they have higher nuclear localization than their mRNA counterparts (Djebali et al. 2012). Whether such observations are a reflection of differences in the transcriptional processing of lincRNAs, and whether differential transcriptional regulation may have functional implications, remains unknown.

At the primary sequence level, most lincRNAs are less evolutionarily conserved than mRNAs (Guttman et al. 2009; Hezroni et al. 2015), leading some to argue that lincRNAs may be functionless by-products of transcriptional noise (Kowalczyk et al. 2012). However, recent work has found that a subset of lincRNAs contain

Corresponding author: john_rinn@harvard.edu

Article published online before print. Article, supplemental material, and publication date are at http://www.genome.org/cgi/doi/10.1101/gr.214205.116. Freely available online through the Genome Research Open Access option. conserved regulatory elements, including transcription factor binding sites (Necsulea et al. 2014), nuclear localization signals (Hacisuleyman et al. 2016), and splicing motifs (Ponjavic et al. 2007; Haerty and Ponting 2015). This suggests that the pre- and post-transcriptional regulation of some lincRNAs is functionally relevant. Despite this, a global assessment of the importance of lincRNA pre- and post-transcriptional regulation, and how such regulation compares to that of mRNAs, remains unresolved.

Here, we combine a battery of functional genomic analysis and biochemical assays to comprehensively interrogate the differences between the life cycles of lincRNAs and mRNAs in humans. Our analysis provides an extensive characterization of the lincRNA life cycle and its distinguishing properties from that of mRNA.

\section{Results}

Active lincRNA promoters are depleted for most histone marks but are enriched in $\mathrm{H} 3 \mathrm{~K} 9 \mathrm{me} 3$

To systematically survey the properties associated with the RNA life cycle, and to avoid potentially confounding influences arising from the regulation of overlapping genes, we focused our analysis on intergenic lncRNAs (termed "lincRNAs"). These lincRNAs were compared to a reference set of mRNAs (Supplemental Methods), resulting in a data set comprising 5196 lincRNAs and 19,575 mRNAs. For most analyses, we only compared lincRNAs to mRNAs with

(C) 2017 Melé et al. This article, published in Genome Research, is available under a Creative Commons License (Attribution-NonCommercial 4.0 International), as described at http://creativecommons.org/licenses/by-nc/4.0/. 
similar expression levels. We first addressed whether the promoters of transcribed lincRNAs (defined as "active promoters") and a set of expressionmatched mRNAs differed in their chromatin environment and transcriptional regulation. We defined promoter regions as $5 \mathrm{~kb}$ upstream of and downstream $( \pm 5$ $\mathrm{kb})$ from the TSS. We then curated chromatin immunoprecipitation (ChIP) experiments for 70 histone modifications and 370 transcription factors, in seven and eleven ENCODE cell lines, respectively (The ENCODE Project Consortium 2004). Finally, we used RNA-seq from 20 human tissues to calculate tissue-specificity (Cabili et al. 2011).

We found that, in each of the seven tested cell lines and relative to their mRNA counterparts, the promoters of most lincRNAs were depleted for nearly all histone marks (Fig. 1A; Supplemental Fig. S1). Intriguingly, the only histone mark enriched in active lincRNA promoters compared to mRNAs was H3K9me3, a modification commonly found in transcriptionally repressed regions (Fig. 1A,B; Supplemental Fig. S2; Supplemental Table S1). Despite this, lincRNA genes with and without H3K9me3 were expressed at similar levels in all but one cell line (Wilcoxon test; FDR $<0.05$ ) (Supplemental Fig. S3). Interestingly, H3K9me3-marked lincRNAs were significantly more tissue-specific than those lacking this histone mark, in five of the seven cell lines $($ FDR $<0.05)$ (Supplemental Fig. S4). In expressionmatched mRNAs, neither expression levels nor tissue specificity differed significantly with regard to $\mathrm{H} 3 \mathrm{~K} 9 \mathrm{me} 3$ status (Wilcoxon test; FDR $>0.05$ ) (Supplemental Figs. S3, S4).

\section{LincRNA promoters are enriched for specific transcription factors and have conserved binding sites}

Using ChIP data, we next examined the binding of transcription factors at lincRNA promoters. In general, lincRNA promoters were bound by fewer classes of TFs than those of expression-matched mRNAs (Fig. 1C; Supplemental Table S2). However, some TFs-including the GATA family, JUN, and FOS-were consistently enriched at lincRNA promoters in all cell lines (Fig. 1D; Supplemental Table S3). To assess the functional relevance of these interactions, we calculated average conservation scores across TF binding sites (TFBSs) that intersected either lincRNA or mRNA promoters. We found that in lincRNA promoters, $87.4 \%$ of TFs had binding sites that were conserved compared to their flanking regions $(\mathrm{FDR}<$

D

F
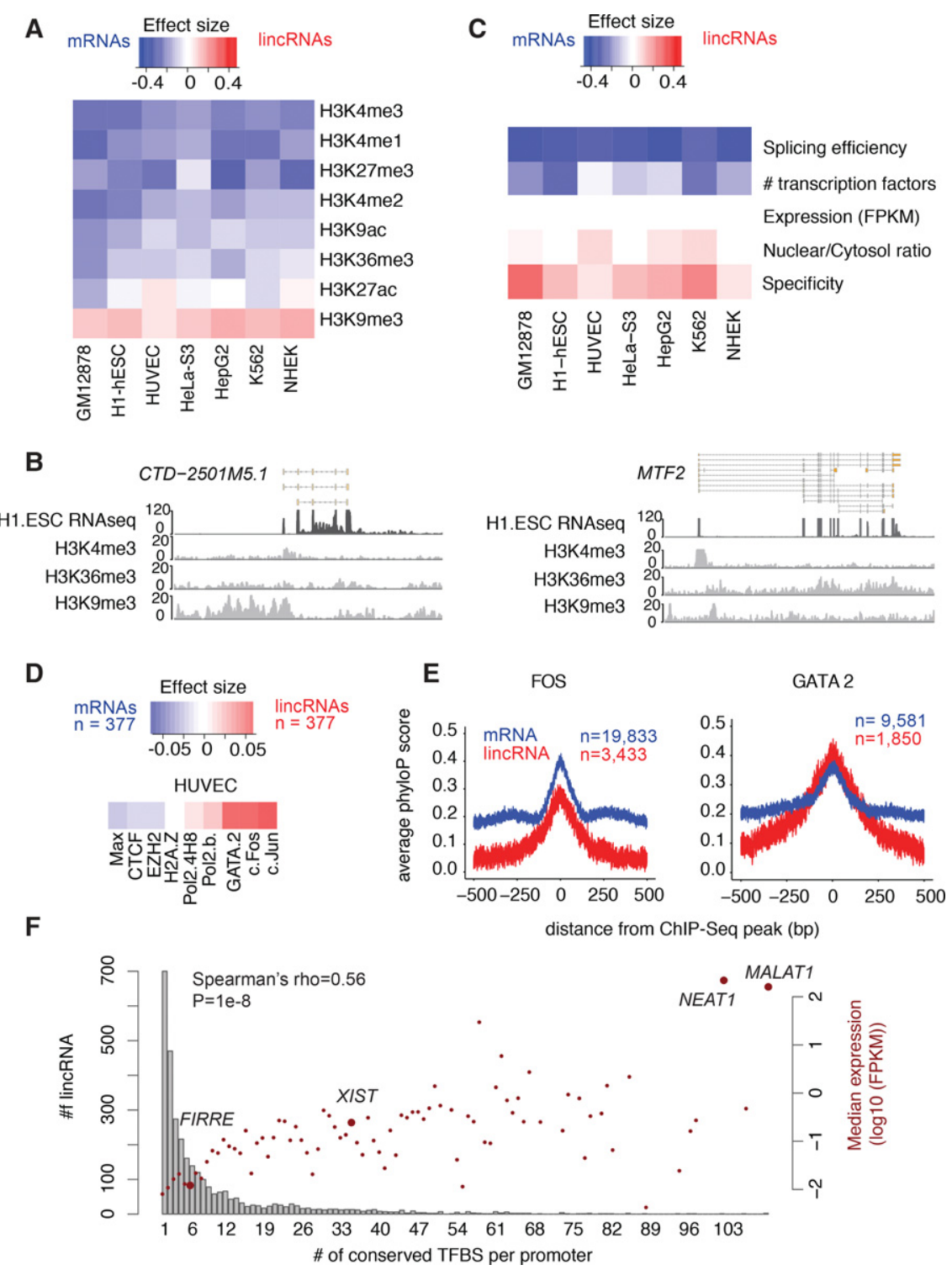

Figure 1. Certain histone marks and TFs are enriched in lincRNA promoters. (A) Fisher effect size differences, comparing the presence or absence of eight histone marks (observed via ChIP) in the promoters of lincRNAs and mRNAs, in seven ENCODE cell lines. Blue corresponds to larger values in mRNAs and red to larger values in lincRNAs. The number of genes ranged from 754 (HUVEC) to 1262 (GM12878) and corresponds to all lincRNAs expressed at $>0.1$ FPKM in the tested cell line, and to expression-matched mRNAs. (B) ChIP-seq read coverage for three histone marks in one lincRNA (left) and one mRNA (right) with similar expression levels in H1 ESCs. (C) Wilcoxon effect size differences between lincRNAs and mRNAs across several gene/promoter properties. The genes analyzed are the same as in $A$. (D) Fisher effect size differences between lincRNA and mRNA promoters comparing the presence or absence of TFs, in HUVEC cells. (E) Average conservation at FOS (left) and GATA2 (right) binding sites overlapping lincRNA and mRNA promoters centered on ChIP-seq peaks. Width of the lines represents the standard error. $(F)$ Histogram of the number of lincRNAs with different numbers of TFs with conserved binding sites in their promoters (bars, left axis) and the corresponding median expression for that group of lincRNAs (dots, right axis). The identity of notable functional lincRNAs within each group is highlighted.

$0.05)$, whereas in mRNA promoters, $97.8 \%$ of TFs were conserved (Supplemental Fig. S5; Supplemental Tables S4, S5). Nevertheless, in some cases, such as for GATA2, KAP1, and MBD4, the average conservation was higher in lincRNA promoters than in mRNA promoters (Fig. 1E; Supplemental Fig. S5). These results were consistent irrespective of how promoters were defined (i.e., in windows of $10 \mathrm{~kb}$ or windows of $3 \mathrm{~kb}$ ) or whether TFBSs

\section{Genome Research}

www.genome.org 
were based on ChIP-seq peaks or on known TF binding motifs (Supplemental Figs. S6, S7).

We next analyzed the conservation of TFBSs in lincRNA promoters, testing whether nucleotides within each TFBS were more conserved than those in the surrounding region (FDR $<0.05$ ). We observed that $61.9 \%$ of lincRNA promoters had at least one conserved TFBS (median of 1 , mean of 6.2 conserved TFBSs per lincRNA promoter) (Fig. 1F; Supplemental Tables S6, S7). Similarly, 65.9\% of the promoters of expression-matched mRNAs had at least one conserved TFBS (median of 2, mean of 8.55 conserved TFBSs per promoter) (Supplemental Fig. S8). These proportions were larger than those found in random intergenic regions (Supplemental Table S8). Furthermore, expression of both lincRNAs and mRNAs increased with the number of conserved TFBSs (Fig. 1F; Supplemental Fig. S8). Conversely, tissue specificity decreased with increasing number of conserved TFBS types (Supplemental Figs. S9, S10). Finally, we found that lincRNAs present in lncRNAdb (a database of functionally characterized lincRNAs, [Amaral et al. 2011]) had significantly more conserved TFBSs than lincRNAs lacking functional characterization (Wilcoxon $\left.P=1.09 \times 10^{-5}\right)$. Together, these results suggest that the number of conserved promoter TFBSs might be a useful characteristic with which to identify functional lincRNAs.

\section{LincRNA splicing is inefficient}

We next sought to systematically investigate splicing efficiency in lincRNAs, which has been previously examined in smaller scale studies (Seidl et al. 2006; Tilgner et al. 2012). To this end, we developed a gene-level metric for calculating splicing efficiency (Supplemental Fig. S11A). Reasoning that differences in splicing might be more evident in the nuclear pool of RNA, we first analyzed fractionated nuclear RNA-seq data from seven ENCODE cell lines (Djebali et al. 2012). Compared to mRNAs with similar expression levels, lincRNAs generally exhibited inefficient splicing in all cell lines (Fig. 2A; Supplemental Table S1; Supplemental Figs. S12A, S13).

Strikingly, splicing efficiency was the most discriminatory feature between lincRNAs and mRNAs (Fig. 1C). As expected, in the cytosolic fraction, the splicing efficiencies of all RNAs were higher, though lincRNAs were still less efficiently spliced than mRNAs (Fig. 2B; Supplemental Fig. S12B). We found no relationship between splicing efficiency and presence of H3K9me3 (FDR $<0.05$ ) (Supplemental Fig. S15). Notably, functionally characterized lincRNAs exhibited greater splicing efficiency than noncharacterized lincRNAs with similar expression levels (Fig. 2C,D;
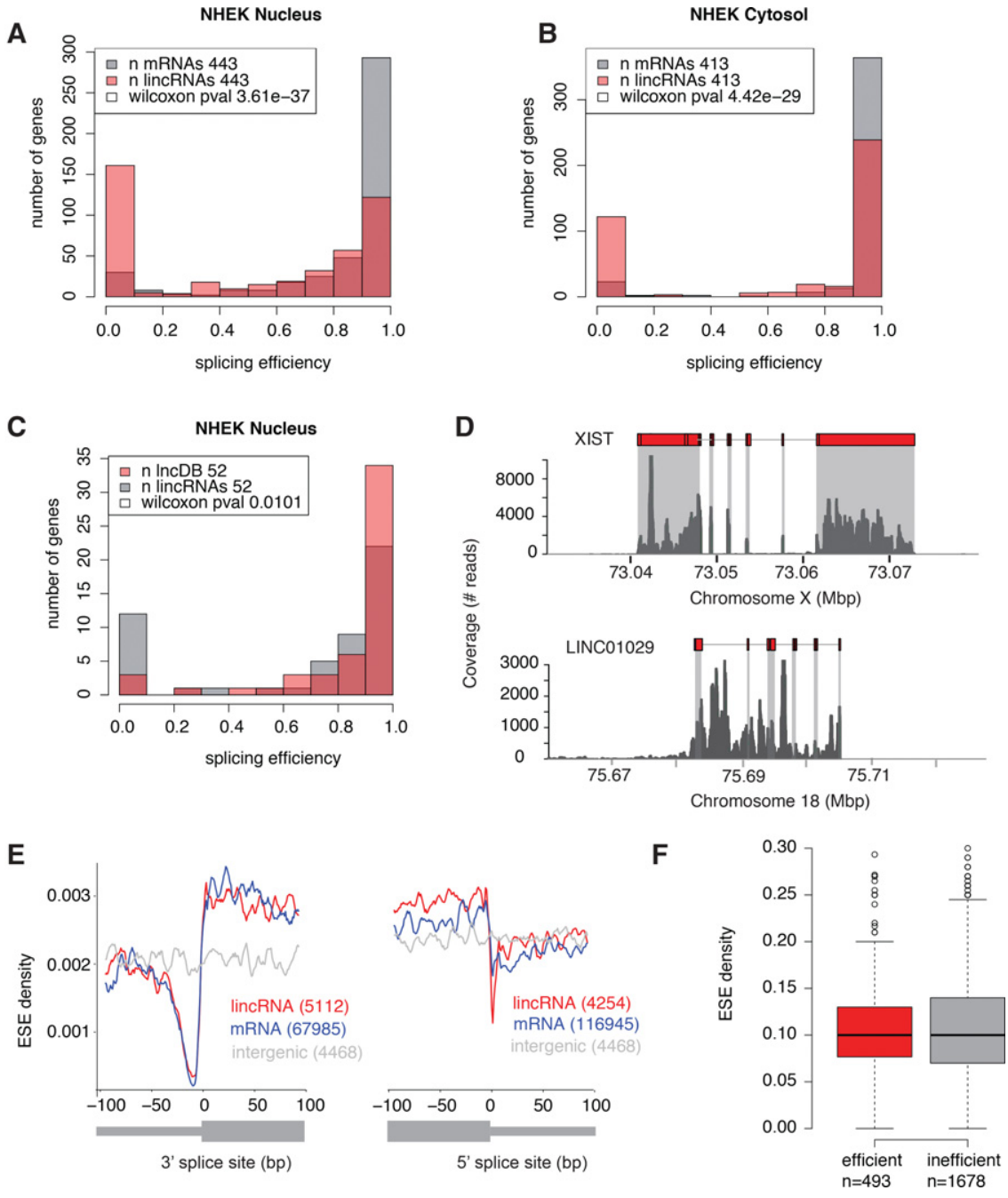
FPKM) and expression-matched mRNAs in the nuclear fraction $(A)$ and cytosolic fraction $(B)$ of human pression-matched $(>0.1 \mathrm{FPKM})$ uncharacterized lincRNAs. $(D)$ RNA-seq read coverage for a representapared to random intergenic regions of the same length. $(F)$ Distribution of ESE density per gene in efficiently $(>0.5)$ and inefficiently spliced $(<0.5)$ lincRNAs.

Supplemental Fig. S14). Thus, perhaps-similarly to the abundance of conserved TFBSs_-splicing efficiency might represent a principal consideration when identifying functional lincRNAs.

To exclude the possibility that the apparent splicing inefficiency derived from inaccuracies in our lincRNA gene models, we repeated our analysis using three different gene sets with more reliable annotations. Specifically, we selected (1) genes annotated as "known" in GENCODE (Supplemental Fig. S16), (2) genes expressed at greater than 1 FPKM (Supplemental Fig. S17), and (3) genes with splicing efficiency larger than 0.1 (Supplemental Fig. S18). In 20 out of the 21 comparisons (three gene sets per seven cell lines), splicing efficiency was lower in lincRNAs, consistent with our initial observation. Indeed, we also observedby performing a similar analysis using nuclear and cytosolic RNA-seq data from mouse embryonic stem cells-that murine 
lincRNAs were less efficiently spliced than control mRNAs (Supplemental Fig. S19). This would suggest that inefficient splicing might be a common feature of lincRNAs across species.

Last, we sought to determine if there might be sequence features that could account for the reduction in lincRNA splicing efficiency. To address this, we determined if there were differences in exonic splicing enhancers (ESEs), sequence motifs located within exons that regulate alternative splicing (Blencowe 2000). ESEs have been shown to be conserved in lincRNAs (Schüler et al. 2014; Haerty and Ponting 2015). We found that ESE density in lincRNAs was higher than in mRNAs, both in human (Fig. 2E) and mouse (Supplemental Fig. S20), and that this could be partially explained by differences in GC content (Supplemental Fig. S21). Efficiently spliced lincRNAs did not have more ESEs than inefficiently spliced lincRNAs (one-tailed Wilcoxon test $P>0.05$ ) (Fig. 2F; Supplemental Fig. S22), indicating that ESE density cannot account for differences in mRNA vs. lincRNA splicing efficiency.

\section{The U1-PAS axis is similar in lincRNAs and mRNAs}

Another possible factor influencing splicing efficiency could be the binding of the U1 small nuclear ribonucleoprotein (that recognizes and binds the $5^{\prime}$ splice site and facilitates the recruitment of the spliceosome to the unspliced transcript). Binding of U1 to nascent RNA transcripts is also thought to occlude alternative polyadenylation signals (PAS), thereby protecting the transcript from degradation and discriminating bona fide transcripts from transcriptional noise (Almada et al. 2013).

We first evaluated whether presence of canonical U1 motifs was related either to splicing efficiency or to lincRNA locus length. In most cell lines, the presence of a canonical U1 site within a lincRNA—either within the first $1 \mathrm{~kb}$ (Fig. 3A; Supplemental Fig.
S23) or within all 5' splice sites (Spearman's rho; FDR >0.05) did not significantly correlate with the efficiency with which that RNA was spliced. However, RNAs with U1 sites within the first $1 \mathrm{~kb}$ were longer than those without, and this difference was greater for lincRNAs than for mRNAs (Fig. 3A).

Next, we used a discriminative hexamer analysis (Almada et al. 2013) to assess whether lincRNAs exhibited the so-called "U1-PAS" axis-an enrichment of U1 binding motifs and depletion of polyadenylation motifs downstream from the TSS. In general, the U1PAS properties of lincRNAs largely mirrored those of their mRNA counterparts. For example, U1 motifs were enriched and polyadenylation motifs were depleted in the sense strand of lincRNA genes, relative to the upstream antisense strand (Fig. 3B). Moreover, U1 motifs were highly enriched in the 200 bp downstream from the TSS in both lincRNAs and mRNAs (Fig. 3C; Supplemental Table S9). Finally, as in mRNAs, lincRNA U1 motifs were more conserved than were nearby sequences; these U1 conservation signals were further strengthened when located at annotated exon-intron junctions (Fig. 3D). We thereby conclude that features of the U1-PAS axis cannot account for inefficient lincRNA splicing.

\section{Internal splicing signals are weaker in lincRNAs than in mRNAs}

Thus far, we have not been able to attribute differences in splicing efficiency between lincRNAs and mRNAs to differences in ESE density, the U1-PAS axis, or the canonical U1 5' splice site. We therefore investigated whether differences in other splicing signals, such as the polypyrimidine tract (PPT) and the branch point (Fig. 4A), might explain this phenomenon. The PPT signals of lincRNAs had a slightly smaller proportion of pyrimidines (Fig. 4B; Supplemental Fig. S24) and a larger ratio of uracils to cytosines (Fig. 4C,D) than those of their mRNA counterparts. In most cell
A

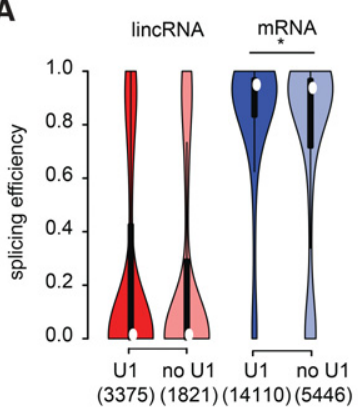

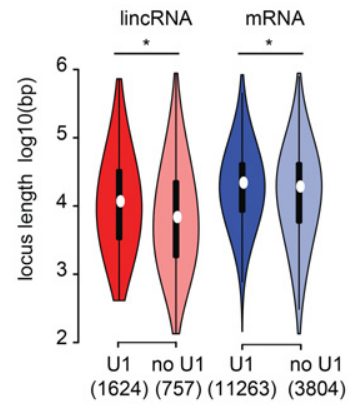

C

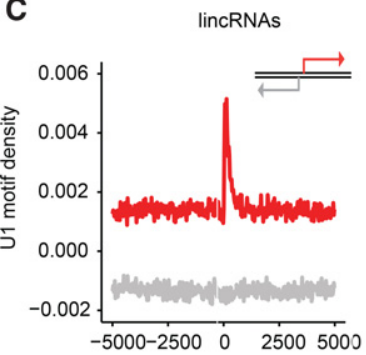

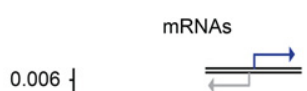

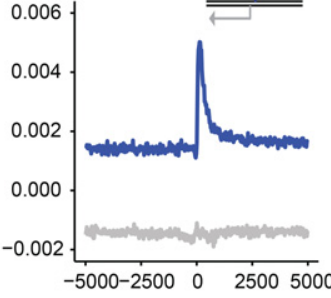

distance to TSS (bp)

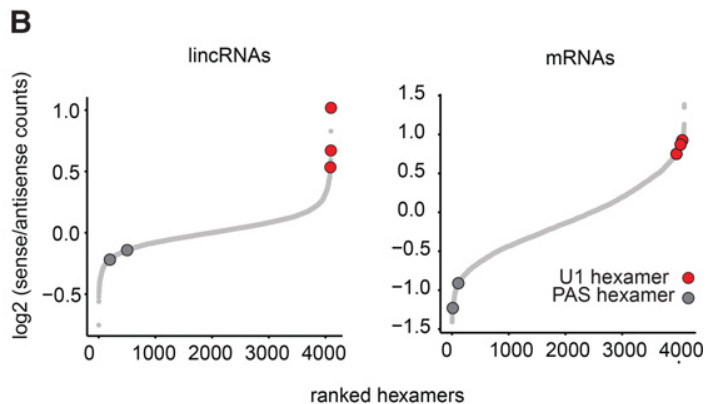

D

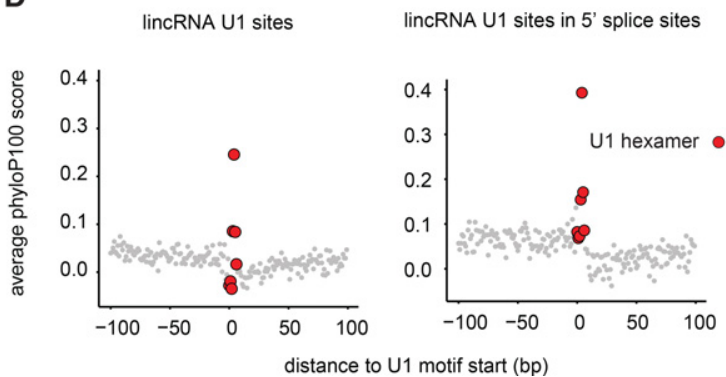

Figure 3. The U1-PAS axis is similar in lincRNAs and mRNAs. ( $A$ ) Distribution of splicing efficiency (left) and locus length (right) in lincRNAs and mRNAs in $\mathrm{K} 562$, with and without canonical U1 motifs at $5^{\prime}$ splice sites within the first $1 \mathrm{~kb}$ downstream from the TSS. Asterisks indicate significant differences (Wilcoxon $P$-value $<0.05)$. (B) Rank of all hexamers by enrichment in the first $1 \mathrm{~kb}$ in the sense direction relative to upstream antisense direction in lincRNAs (left) and mRNAs (right). (C) U1 motif density around the TSS in lincRNAs (left) and mRNAs (right) in the sense strand (red or blue) and in the antisense strand (gray). (D) Average conservation at all U1 nucleotides present in the first $1 \mathrm{~kb}$ of lincRNAs (left) or the subset of these U1 nucleotides that overlap 5' splice donors (right).

\section{Genome Research}

www.genome.org 
A

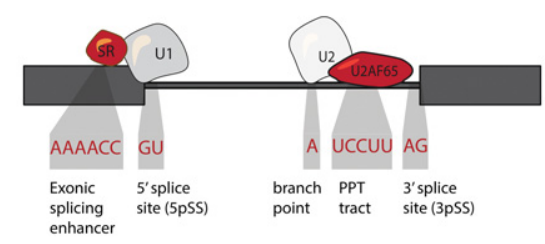

B

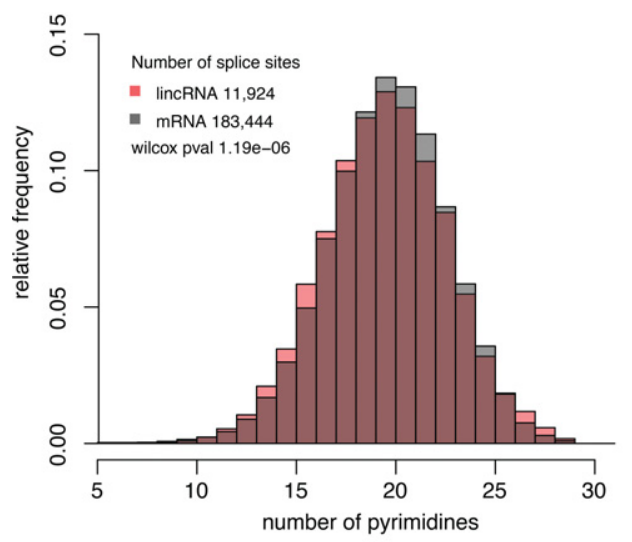

C

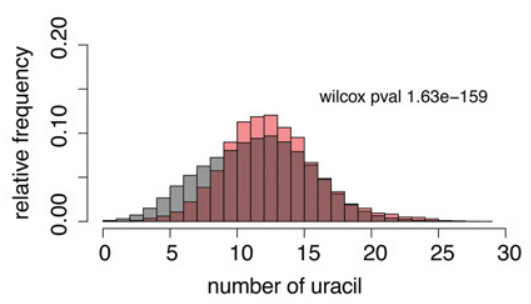

D

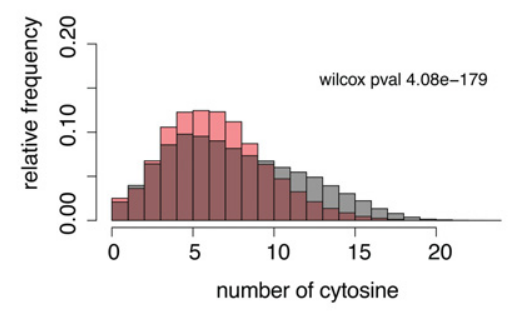

E

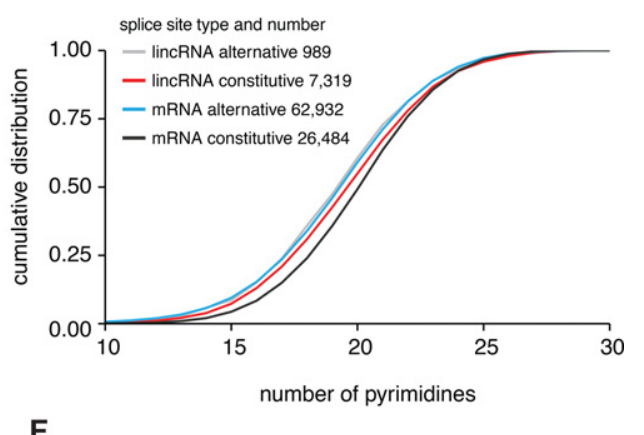

F

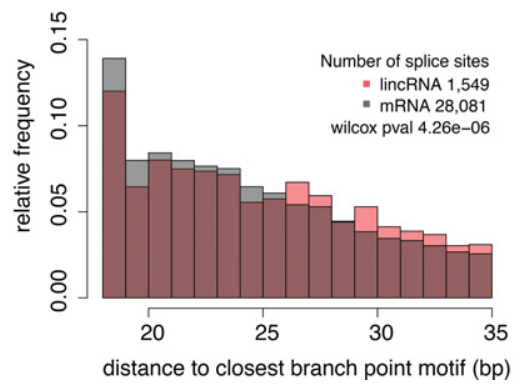

Figure 4. Internal splicing motifs are slightly weaker in lincRNAs than in mRNAs. (A) Schematic view of necessary splicing RNA motifs and splicing regulators. $(B)$ Relative frequency of pyrimidine nucleotides upstream of the $3^{\prime}$ splice sites. $(C, D)$ Relative frequencies of uracil $(C)$ and cytosine $(D)$ nucleotides upstream of the $3^{\prime}$ splice site. Number of $3^{\prime}$ splice sites analyzed is the same as in $B$. (E) Relative frequency of pyrimidine nucleotides upstream of the $3^{\prime}$ splice site grouped by splice-site type (alternative or constitutive). ( $F$ ) Distribution of distances (bp) between $3^{\prime}$ splice site and the nearest canonical branch point motif. (wilcox pval) Wilcoxon $P$-value.

lines, considering both mRNAs and lincRNAs, the overall number of pyrimidines positively correlated with splicing efficiency (Supplemental Fig. S25). Moreover, constitutive splice sites exhibited a larger proportion of pyrimidine nucleotides than did those of alternatively spliced sites in both lincRNAs and mRNAs (Fig. 4E).

We next investigated the distance between the branch point and 3' splice sites, which has been previously shown to affect splicing efficiency (Rosenberg et al. 2015). These distances were greater in lincRNAs than in mRNAs, as gauged both by in silico mapping the canonical branch motif (Fig. 4F; Supplemental Fig. S26) and as observed in CaptureSeq data targeting splicing branch points (Supplemental Fig. S27; Mercer et al. 2015). In summary, the number of pyrimidines and branch point differences within internal $3^{\prime}$ splice sites could account for some of the splicing differences between lincRNAs and mRNAs.

Binding of U2AF65 and splice site conservation correlates with greater splicing efficiency in lincRNAs

Based on the above observations, we hypothesized that weak internal 3' splice-site signals may contribute to the lower splicing efficiency of lincRNAs. One important factor in splice site determi- nation is U2AF65, which binds to the PPT and promotes binding of the U2 snRNP to the branch point (Fig. 4A). Consistent with the known role of U2AF65, we observed that U2AF65 peaks were enriched near 3' splice sites in both lincRNAs and mRNAs in two publicly available U2AF65 CLIP-seq data sets (Fig. 5A,B; Zarnack et al. 2013; Shao et al. 2014). While U2AF65 binding was depleted in lincRNAs relative to mRNAs in both data sets (Fig. 5A,B), lincRNAs and mRNAs with similar expression levels exhibited similar peak densities (Supplemental Fig. S28).

To address whether U2AF65 binding was related to splicing efficiency, we compared the splicing efficiencies of expressionmatched lincRNAs that were or were not bound by U2AF65. In both CLIP data sets, U2AF65-bound lincRNAs exhibited significantly greater splicing efficiencies than their unbound counterparts (one tailed Wilcoxon $P<0.05$ ) (Fig. 5C,D). We observed similar results for lincRNAs with one 3' splice site (Supplemental Fig. S29A), or when lincRNAs with zero splicing efficiency were excluded from analysis (Supplemental Fig. S29B). Likewise, U2AF65bound mRNAs were also more efficiently spliced than unbound mRNAs (one tailed Wilcoxon $P<0.05$ ) (Supplemental Fig. S30). Thus, less U2AF65 binding may contribute to poor splicing in lincRNAs. 
A

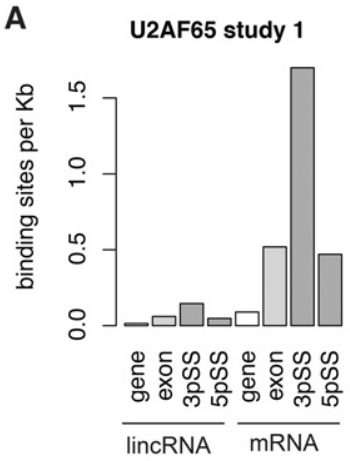

B

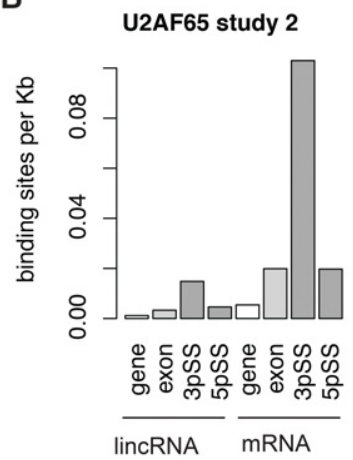

C

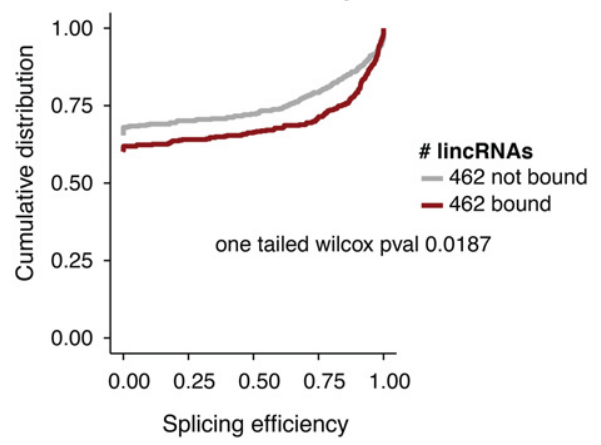

D

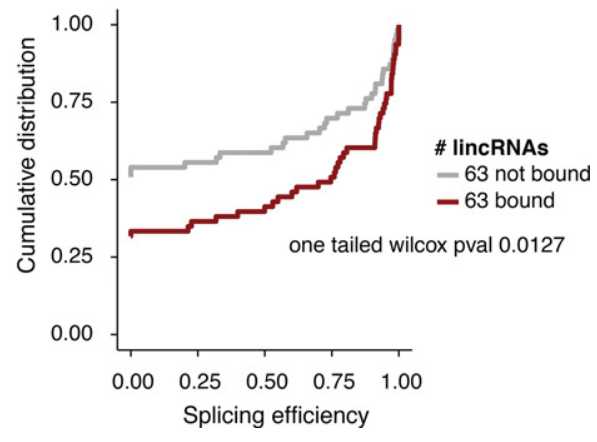

Figure 5. U2AF65 binding in lincRNAs and mRNAs. $(A, B)$ U2AF65 CLIP-seq binding site density in lincRNAs (left) and mRNAs (right), quantified across the entire gene locus, the processed transcript (exons), and the $3^{\prime}$ and $5^{\prime}$ splice sites. Splice sites were defined as \pm 50 bp from any splice junction. $(C, D)$ Cumulative distribution of splicing efficiency comparing U2AF65-bound or expression-matched unbound lincRNAs. Study 1 corresponds to data from Zarnack et al. (2013) and study 2 to Shao et al. (2014).

We next examined the relationship between lincRNA splicing efficiency and the conservation of $5^{\prime}$ and $3^{\prime}$ splice sites (Supplemental Fig. S31). On average, both $5^{\prime}$ and $3^{\prime}$ splice junctions were significantly conserved in lincRNAs (Empirical $P<$ 0.011 and $P<0.005$, respectively). Moreover, 1105 lincRNAs (21\%) had at least one significantly conserved (5' or $\left.3^{\prime}\right)$ splice site (Supplemental Table S9). These lincRNAs were more efficiently spliced than those lacking conserved sites, even when correcting for expression levels (Supplemental Fig. S32). Furthermore, the set of lincRNAs with conserved splice sites $\left(5^{\prime}\right.$ or $\left.3^{\prime}\right)$ was enriched for functionally characterized lincRNAs (Fisher's exact test $P=$ $1.7 \times 10^{-5}$ and $P=0.034$, respectively), indicating that splice site conservation may provide a further metric by which to computationally identify functional lincRNAs.

\section{LincRNAs and mRNAs exhibit similar stability}

To complete our systematic survey of the properties associated with the lincRNA life cycle, we next focused on RNA decay. LincRNAs have been previously observed to be less stable than mRNAs (Clark et al. 2012), although an analysis that incorporates expression levels into assessments of stability has not yet been performed. To address this, we systematically measured the half-lives of lincRNAs and mRNAs following actinomycin D treatment (Methods) in two human cell lines: K562 and human embryonic stem cells (HUES9). Strikingly, when we normalized for pretreatment expression levels, we found that the half-lives of lincRNAs were indistinguishable from those of mRNAs in both cell lines

(one tailed Wilcoxon $P>0.05$ ) (Fig. 6A; Supplemental Fig. S33; Supplemental Table S10).

Furthermore, sequence elements that correlated with RNA stability were similar among lincRNAs and expressionmatched mRNAs. We examined the nucleotide composition of 7-mers that were significantly different between stable and unstable transcripts. Sequences depleted at the $5^{\prime}$ end of all stable transcripts (lincRNAs and mRNAs) were CG rich, while those at the $3^{\prime}$ end were $\mathrm{AU}$ rich, in both cell lines (Fig. 6B; Supplemental Fig. S34). However, the specific $k$-mers that were enriched in lincRNAs and mRNAs differed (Supplemental Figs. S35, S36). In addition, in HUES9 cells, low complexity repeats were enriched in both unstable lincRNAs and mRNAs, while in $\mathrm{K} 562$ cells this enrichment was only observed in unstable mRNAs (Wilcoxon test $P=0.002$ ) (Fig. 6C; Supplemental Figs. S37, S38).

Finally, we assessed how HuR (encoded by ELAVL1), a known stability regulator that binds to RNA transcripts, may influence lincRNA half-life, using CLIPseq data (Kishore et al. 2011). We found that, while HuR preferentially bound near the $3^{\prime}$ ends of mRNAs, such positional bias did not exist in lincRNAs regardless of whether the comparison was for expression-matched transcripts (Supplemental Fig. S39) or not (Fig. 6D; Supplemental Fig. S40).

\section{Discussion}

Here, we have provided a comprehensive characterization of the pre-, co-, and post-transcriptional regulation properties of lincRNAs relative to those of mRNAs. By systematically surveying key aspects of the lincRNA life cycle-from synthesis to degradation-and comparing them to equivalent stages of the mRNA life cycle, we have elucidated many features that distinguish these two classes of RNA (Fig. 7). In addition, we have identified characteristic signatures that are enriched in known functional lincRNAs.

The first set of properties that distinguish lincRNAs from mRNAs relates to histone modifications and promoter regulation. Previous reports have suggested that transcribed lincRNAs and mRNAs had similar histone marks (Guttman et al. 2009). Whereas in general terms lincRNAs and mRNAs share similar histone marks, such as H3K4me3 in their promoter and H3K36 in the gene body, when comparing expression-matched populations, there are slight differences. Possibly the most striking difference is that $\mathrm{H} 3 \mathrm{~K} 9 \mathrm{me}$ - $-\mathrm{a}$ canonically repressive histone mark-is enriched in the promoters of active lincRNAs (Alam et al. 2014) compared to active mRNAs, and that expression of H3K9me3-marked lincRNAs is more tissue-specific than that of lincRNAs lacking H3K9me3. In addition, lincRNAs have fewer transcription factors bound to their promoters than similarly expressed mRNAs. Since our analysis controlled for RNA expression levels, these observations are likely to reflect fundamental differences in the regulation

\section{Genome Research}

www.genome.org 
A
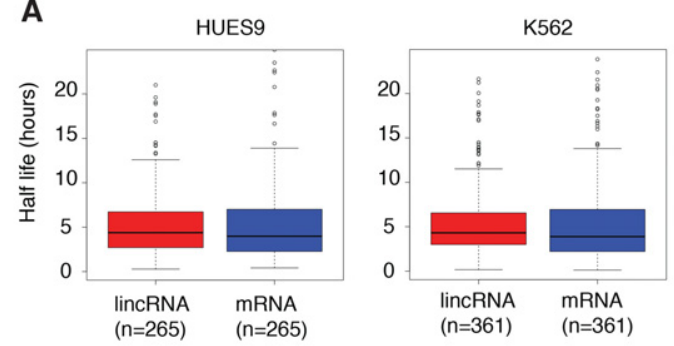

B

k-mer composition depleted in stable transcripts

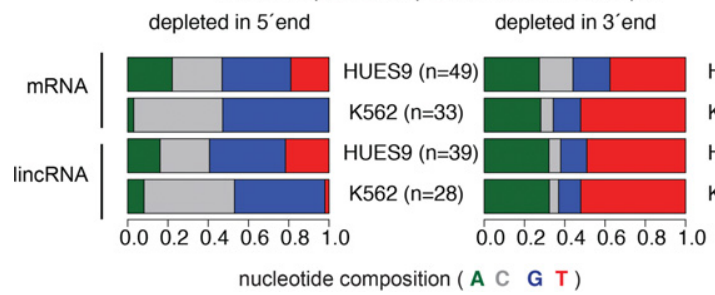

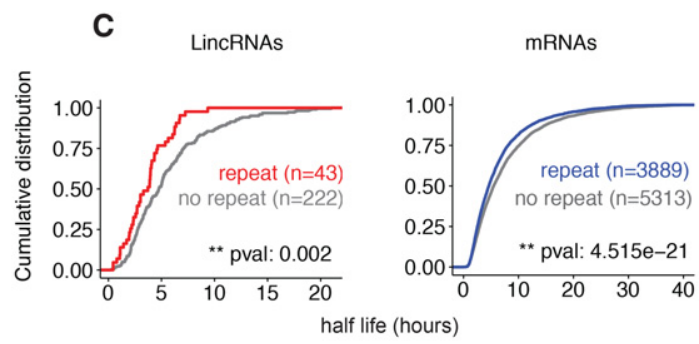

D

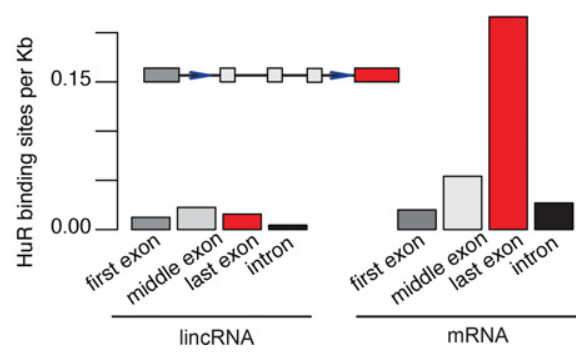

Figure 6. LincRNAs are as stable as expression-matched mRNAs. ( $A$ ) Half-lives of expression-matched lincRNAs (red) and mRNAs (blue) after actinomycin treatment in HUES9 (left) and K562 (right) cells. (B) Nucleotide composition of 7-mers that are depleted in stable mRNAs and lincRNAs in both the $5^{\prime}$ (left) or $3^{\prime}$ ends (right). 7 -mers were called as significant at FDR $<0.05$ for mRNAs and at $P$-values $<0.05$ for lincRNAs, due to their low sample size. ( $C$ ) Cumulative distribution of half-lives for lincRNAs (left) and mRNAs (right) in HUES9 with and without at least one exon that intersects low complexity repeats. ( $D$ ) Density of HuR binding sites in different regions of lincRNA (left) and mRNA (right) transcripts.

of lincRNA promoters. Whereas mRNAs may require relatively high numbers of TFs to maintain baseline levels of expression across many tissues, lincRNA promoters may generally be in a more repressed state and are only activated in certain tissues or at certain developmental time points.

Earlier studies suggested that lincRNA promoters are more conserved than mRNA promoters (Carninci et al. 2005), although later work has reported the opposite (Kutter et al. 2012). Here, we find that lincRNA promoters contain many conserved transcription factor binding sites. In fact, the majority of lincRNA loci have at least one conserved TFBS, suggesting that TF binding at lincRNA promoters is functionally relevant. Furthermore, in some instances, these TFBSs are more conserved in lincRNAs than in mRNAs. For example, GATA2, one of the main regulators of hematopoiesis, is more enriched and conserved in lincRNA promoters than in mRNA promoters. Additionally, the number of conserved TFBSs is greater in functionally characterized lincRNAs, suggesting that regulation at the promoter level should be taken into account when selecting candidate lincRNAs for follow-up studies.

The most distinctive feature of lincRNA biogenesis pertains to the efficiency and regulation of splicing (Fig. 1C). Previous studies suggested that lincRNAs were inefficiently spliced (Tilgner et al. 2012) but had not investigated potential causes of this inefficiency. We have found that lincRNAs have slightly weaker splicing determinants, such as branch point position and PPT sequence composition, and lower binding of the splicing factor U2AF65. It will be intriguing to see if this observation is paralleled in the binding profiles of other core or auxiliary splicing factors. Moreover, this suggests that splicing may not be required for a substantial proportion of lincRNAs and that the annotations of many lincRNAs might require additional unspliced or partially spliced isoforms. One of the lincRNAs that was first identified as being inefficiently spliced was Airn, for which the act of transcription, rather than the RNA molecule itself, has since been shown to be the biologically functional unit (Latos et al. 2012).
However, we also identified a substantial proportion of lincRNAs that are efficiently spliced with conserved splice junctions. Interestingly, efficient splicing is more prevalent among lincRNAs that have been shown to have specific functions, such as XIST (Cerase et al. 2015), FIRRE (Hacisuleyman et al. 2014), and MIAT (Liao et al. 2016). Indeed, efficient splicing has been shown to be necessary for some lincRNAs to carry out their function (Marquardt et al. 2014). Therefore, our results suggest that efficient splicing is an important step in the processing of a subset of lincRNAs, perhaps those that play important roles as RNAs within the cell.

While our analysis has revealed many distinctive characteristics of lincRNA biogenesis, other aspects of post-transcriptional processing have been demonstrated to be similar between lincRNAs and mRNAs. First, canonical U1 sites are as common in lincRNAs as in mRNAs. This finding differs from the observation that lncRNAs arising from divergent mRNA promoters have fewer U1 sites than their mRNA counterparts (Almada et al. 2013) and suggests that lincRNAs and divergent lncRNAs have different properties. Of interest, lincRNAs with U1 sites are longer than those without such sites, implying that U1 binding is likely to protect nascent lincRNA transcripts from premature degradation, as has been shown for mRNAs (Almada et al. 2013). Furthermore, we find that stabilities of lincRNAs resemble those of expression-matched mRNAs. The only feature discriminating these two classes of molecules pertained to the position-and not the frequency-of stability regulator binding.

Our data support a model wherein the degree of lincRNA post-transcriptional regulation is extremely variable. On one end of the distribution, we find lowly expressed, inefficiently spliced lincRNAs with relatively few conserved promoter TFBSs. Despite having RNA regulatory motifs such as U1, we find no evidence of these lincRNA transcripts being under evolutionary constraint. On the other end, we find highly regulated lincRNAs with efficient splicing, conserved exon-intron junctions, and promoters with several conserved TFBSs. Whereas the former could be the result 
LincRNAs

A

Histone

Marks
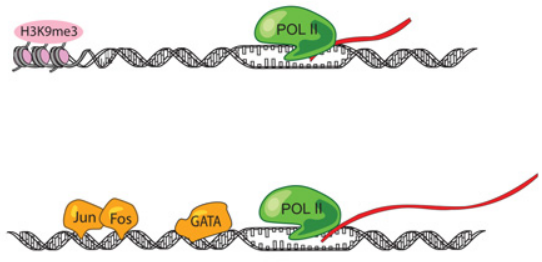

factors

B Transcription

C U1-PAS axis

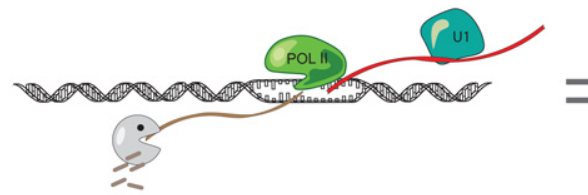

D Splicing efficiency

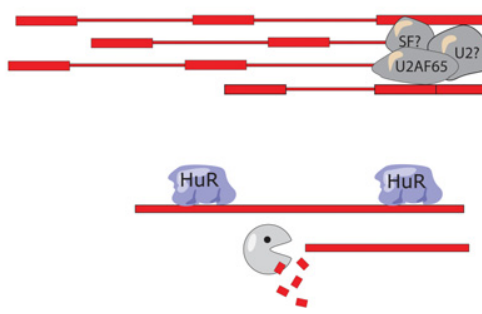

mRNAs
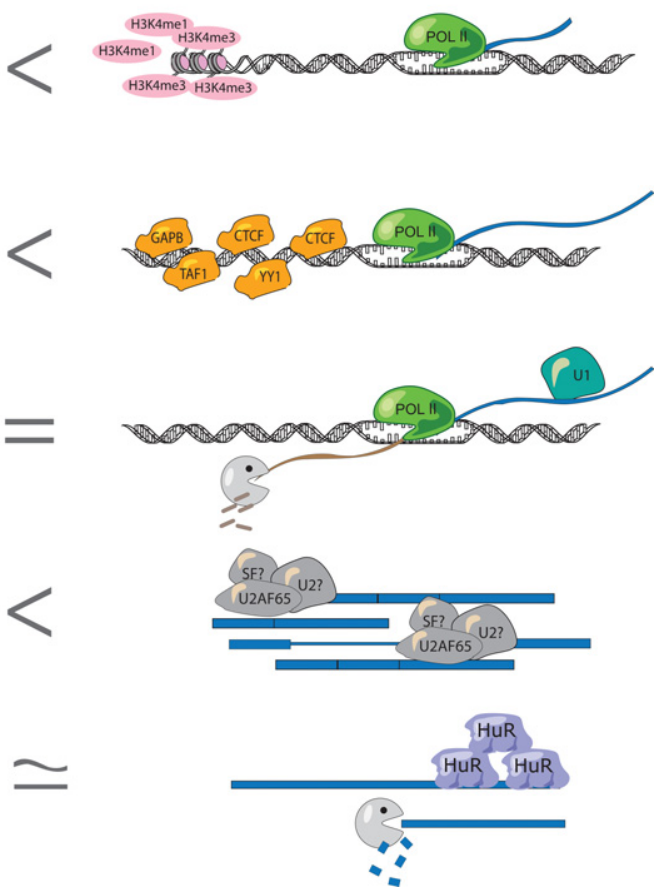

Figure 7. Summary of the similarities and differences between the lincRNA and mRNA life cycles. $(A, B)$ LincRNAs have fewer histone marks $(A)$ and transcription factors (B) bound in their promoter than mRNAs. (C) U1 motif profiles are similar in lincRNAs and mRNAs. (D) Splicing efficiency is lower in lincRNAs than in mRNAs and lincRNAs are depleted for U2AF65-binding. (E) LincRNA and mRNA stability is similar when comparing expression-matched groups. HuR binds equally to both classes but is biased toward 3' UTR localization in mRNAs alone.

of transcriptional noise or the by-product of a necessary act of transcription (Melé and Rinn 2016), the latter group of more regulated lincRNAs are better candidates to function as RNA molecules. Here, we provide a list of these tightly regulated lincRNAs for future analysis and validation (Supplemental Table S11).

Collectively, these observations reveal important insights into how lincRNA biogenesis is regulated and provide several possible explanations for the differences between lincRNAs and mRNAs-all of which can be tested experimentally. Moreover, this work highlights the substantial variability of transcriptional regulation across lincRNAs and provides novel criteria with which to select candidate functional lincRNAs for future validation.

\section{Methods}

\section{LincRNA and mRNA selection in human and mouse}

We analyzed all genes in the human GENCODE v19 catalog with a biotype of "lincRNA" or "protein_coding" (Harrow et al. 2012). We excluded lincRNAs that were located within $5 \mathrm{~kb}$ of any protein coding gene, and any protein coding genes whose promoter $( \pm 5$ $\mathrm{kb}$ of TSS) overlapped a lincRNA promoter. We also excluded any annotated lincRNAs with protein-coding potential greater than zero according to PhyloCSF (Lin et al. 2011). Transcript-level analyses were performed on each gene's longest transcript. For mouse, we analyzed all genes in the GENCODE vM6 with a biotype of "lincRNA" or "protein_coding." We ignored all lincRNAs with protein coding probability greater than 0.44 according to CPAT (Wang et al. 2013). To select a set of lincRNAs with validated func- tions, we selected those lincRNAs with an Ensembl gene_id in lncRNAdb (Amaral et al. 2011; www.lncRNAdb.com).

\section{ENCODE expression quantification}

To quantify gene expression, we downloaded publicly available RNA-seq data from 11 ENCODE cell lines (GSE30567; poly(A) ${ }^{+}$, whole-cell, nucleus, and cytosol samples: A549, GM12878, H1hESC, HeLa-S3, HepG2, HUVEC, IMR90, K562, MCF-7, NHEK, SK-N-SH). We mapped reads to the human genome (hg19) using TopHat v2.1.0 (Trapnell et al. 2009; Kim et al. 2013) with the "-no-coverage-search" flag and using GENCODE v19 annotation. We quantified expression with Cuffdiff2 (Trapnell et al. 2012). We took a gene or transcript's expression level as the average FPKM level across replicates. We calculated nuclear/cytosolic ratio by dividing the gene expression levels (as fragments per kilobase per million reads, FPKM) in each cell compartment. We considered any gene to be expressed in a particular cell line if its expression levels were $>0.1$ FPKM, similar to what has been used in other large-scale transcriptomics studies (Melé et al. 2015).

\section{Tissue specificity}

We used 16 tissues from the Illumina Human Body Map 2.0 (GSE30611; Adipose, Adrenal, Brain, Breast, Colon, Heart, Kidney, Liver, Lung, Lymph Node, Ovary, Prostate, Skeletal Muscle, Testes, Thyroid, White Blood Cells) and four tissues from Cabili et al. (2011) (GSE30554; Foreskin Fibroblast, HeLa, Lung Fibroblast, Placenta). We mapped and quantified the raw reads as described above. We then defined an entropy-based

\section{Genome Research}

www.genome.org 
"tissue specificity score" for each gene as described previously (Cabili et al. 2011).

\section{ChIP-seq analysis}

We used publicly available ChIP-seq data from ENCODE (www. encodeproject.org). We downloaded broadPeak and narrowPeak files for 91 cell lines from UCSC (Supplemental Table S12) and selected the first replicate from each experiment. We defined promoters as the regions \pm 5000 or $-2000 /+1000$ bp of a TSS. For each TF or histone modification, we used BEDTools (Quinlan and Hall 2010) to intersect the ChIP peaks with the promoters and summed up the number of peaks overlapping each gene's promoter.

\section{Splicing efficiency}

We estimated transcript and gene abundances using a modified annotation that contained an additional isoform per gene spanning the gene locus. We calculated splicing efficiency as the sum of abundances of all annotated isoforms divided by the sum of abundances of all isoforms including the spanning one (Supplemental Fig. S11A). We removed single-exon genes from the analysis. For analysis relying on splice site annotation and to avoid spurious results due to misannotations, we replicated our analyses excluding those lincRNAs that had splicing efficiency equal to zero in cell lines for which splicing efficiency could be calculated.

\section{Nuclear fractionation of mouse ES cells}

We isolated cytoplasmic and nuclear fractions from N2 mESC cells as described (Hacisuleyman et al. 2014) for mESC cells. We prepared poly $(\mathrm{A})^{+}$mRNA-seq libraries using the TruSeq RNA sample preparation kit, v2 (Illumina) as described (Goff et al. 2015). Details of the protocol can be found in Supplemental Methods.

\section{Effect size calculation}

For continuous values, we used Wilcoxon's effect size formula:

$$
\text { Wilcoxon Effect Size }=\frac{Z}{\sqrt{n_{x}+n_{y}}},
$$

where $Z$ is the test statistic and $n$ is the number of observations in the $x$ and $y$ groups that we are comparing. To assess effect size on binary properties such as presence/absence of specific transcription factors or histone marks bound, we used the phi coefficient from the psych package (function: phi) (R Core Team 2014).

\section{Expression matching}

We used the R package matchIt with default settings (Ho et al. 2011) where, for each lincRNA, the mRNA with the closest expression value is selected.

\section{Conservation analyses}

We used the vertebrate 100-way alignment PhyloP scores (Pollard et al. 2010) and extracted the scores from genomic regions using bwtool (Pohl and Beato 2014).

\section{TF conservation}

For each TF, we centered the ChIP-seq peaks overlapping a lincRNA or mRNA promoter at the peak maxima and merged any peaks closer than $50 \mathrm{bp}$. We then compared the 200-bp region centered on the peak maxima to the 100-bp regions at each side and at a dis- tance of $150 \mathrm{bp}$ from the peak as background. To test conservation at single ChIP-seq peaks, we performed the same test for each peak individually. To compare ChIP-seq peaks overlapping a known TF motif, we mapped known motifs using FIMO (Grant et al. 2011) and intersected these with corresponding ChIP peaks using BEDTools.

\section{U1 sites, $3^{\prime}$ and $5^{\prime}$ splice site conservation}

We compared the average score of canonical U1 sites, $3^{\prime}$ and $5^{\prime}$ splice site $k$-mers to all other adjacent $k$-mers in a 200-nt window. We considered a $k$-mer to be conserved if it had a greater average conservation score than $95 \%$ of all other adjacent $k$-mers. We also performed the same test for each sequence individually. We used 2-mers for $3^{\prime}$ splice sites, and 10-mers for $5^{\prime}$ splice sites to account for all potential U1 hexamers in splice donor sites.

\section{Motif analyses}

\section{TF binding motifs}

We downloaded all TF binding motifs from the JASPAR CORE database (Mathelier et al. 2016). We mapped them to the corresponding ChIP-seq peaks that overlapped lincRNA or mRNA promoters using the FIMO program (Grant et al. 2011) from the MEME package (Bailey et al. 2009).

\section{ESES}

We downloaded ESE motifs for both humans and mice from Fairbrother et al. (2002). To calculate ESE density, we used all annotated unique exon-intron junctions in both lincRNAs and mRNAs, where the exons were at least $200 \mathrm{nt}$ long.

\section{U1/PAS}

We used three canonical U1 sites (GGUAAG, GGUGAG, GUGAGU) and two PAS motifs (AAUAAA, AUUAAA) as in Almada et al. (2013).

\section{Polypyrimidine tract}

We counted the number of pyrimidine nucleotides (cytosine or uracil) in a region of $30 \mathrm{nt}$ upstream of all annotated $3^{\prime}$ splice sites.

\section{Branch point}

We mapped the canonical branch point sequence (CU[AG]A[CU]) to the region between every annotated $3^{\prime}$ splice site and $40 \mathrm{bp}$ upstream.

\section{3 ' splice sites}

We selected all annotated nonredundant $3^{\prime}$ splice sites in lincRNAs and mRNAs. We defined constitutive or alternative $3^{\prime}$ splice sites as those present in at least $75 \%$ or in $<25 \%$ of all annotated isoforms of a gene, respectively.

\section{k-mer analyses}

To perform discriminative $k$-mer enrichment analyses, we used the program Jellyfish (Marçais and Kingsford 2011). For each $k$ mer, we then calculated the $\log _{2}$ fold ratio between sequences of interest: either $k$-mers enriched in the downstream sense direction of a TSS as compared to the upstream antisense direction or $k$-mers enriched in stable versus unstable transcripts (details in Supplemental Methods). 


\section{RNA stability assay}

We treated K562 and human embryonic stem cells (HUES9) with actinomycin $\mathrm{D}(5 \mu \mathrm{g} / \mathrm{mL}$ of culture medium $)$ and harvested the cells at 0 and $30 \mathrm{~min}$, and 2, 4, and $8 \mathrm{~h}$ for RNA isolation and measured global RNA levels by poly(A) ${ }^{+}$RNA-seq in triplicate. For each gene, we normalized abundance to GAPDH and fitted the data to a first order exponential decay curve. We then calculated the average half-life per gene across replicates in which the Pearson correlation between the fitted curve and the real curve was higher than 0.7.

\section{CLIP-seq data analysis}

We downloaded CLIP-seq from publicly available data for U2AF65 from HeLa cells from two data sets: Zarnack et al. (2013) (ArrayExpress accession numbers for the iCLIP data: E-MTAB1371) and Shao et al. (2014) (Gene Expression Omnibus GSE61603). We used an established bioinformatics pipeline designed to find CLIP-seq peaks relative to the transcript abundances (Kelley et al. 2014) and calculated peak density across different transcript regions (further details in Supplemental Methods).

\section{Data access}

The sequencing data from this study have been submitted to the NCBI Gene Expression Omnibus (GEO; http://www.ncbi.nlm. nih.gov/geo/) under accession number GSE80046.

\section{Acknowledgments}

We thank David Kelley from the Rinn lab for feedback on CLIP-seq analysis and comments on the manuscript, James Lee for comments on the manuscript, Alex Meissner for providing the HUES9 cells, and Veronica Akopian for technical support with HUES9 culture. M.M. is a Gilead Fellow of the Life Sciences Research Foundation. This work was supported by US National Institutes of Health grants R01 ES020260, R01 MH102416, and P01 GM099117 and by the National Science Foundation Graduate Research Fellowship under Grant No. DGE1144152.

Author contributions: M.M. and J.R. designed the project and wrote the manuscript. K.M. performed all $k$-mer and conservation analysis. W.M. processed ENCODE RNA-seq and ChIP-seq data sets. D.S. performed the fractionation protocol for mouse ES cells. C.G. performed the stability assay. M.M. performed all the remaining analysis. All authors have read and approved the manuscript for publication.

\section{References}

Alam T, Medvedeva YA, Jia H, Brown JB, Lipovich L, Bajic VB. 2014 Promoter analysis reveals globally differential regulation of human long non-coding RNA and protein-coding genes. PLoS One 9: e109443.

Almada AE, Wu X, Kriz AJ, Burge CB, Sharp PA. 2013. Promoter directionality is controlled by U1 snRNP and polyadenylation signals. Nature 499: 360-363.

Amaral PP, Clark MB, Gascoigne DK, Dinger ME, Mattick JS, 2011. lncRNAdb: a reference database for long noncoding RNAs. Nucleic Acids Res 39: D146-D151.

Bailey TL, Boden M, Buske FA, Frith M, Grant CE, Clementi L, Ren J, Li WW Noble WS. 2009. MEME SUITE: tools for motif discovery and searching. Nucleic Acids Res 37: W202-W208.

Blencowe BJ. 2000. Exonic splicing enhancers: mechanism of action, diversity and role in human genetic diseases. Trends Biochem Sci 25: 106-110.

Cabili MN, Trapnell C, Goff L, Koziol M, Tazon-Vega B, Regev A, Rinn JL. 2011. Integrative annotation of human large intergenic noncoding RNAs reveals global properties and specific subclasses. Genes Dev 25: 1915-1927.
Carninci P, Kasukawa T, Katayama S, Gough J, Frith MC, Maeda N, Oyama $\mathrm{R}$, Ravasi T, Lenhard B, Wells C, et al. 2005. The transcriptional landscape of the mammalian genome. Science 309: 1559-1563.

Cerase A, Pintacuda G, Tattermusch A, Avner P. 2015. Xist localization and function: new insights from multiple levels. Genome Biol 16: 166.

Clark MB, Johnston RL, Inostroza-Ponta M, Fox AH, Fortini E, Moscato P, Dinger ME, Mattick JS. 2012. Genome-wide analysis of long noncoding RNA stability. Genome Res 22: 885-898.

Derrien T, Johnson R, Bussotti G, Tanzer A, Djebali S, Tilgner H, Guernec G, Martin D, Merkel A, Knowles DG, et al. 2012. The GENCODE v7 catalog of human long noncoding RNAs: analysis of their gene structure, evolution, and expression. Genome Res 22: 1775-1789.

Djebali S, Davis CA, Merkel A, Dobin A, Lassmann T, Mortazavi A, Tanzer A, Lagarde J, Lin W, Schlesinger F, et al. 2012. Landscape of transcription in human cells. Nature 489: 101-108.

The ENCODE Project Consortium. 2004. The ENCODE (ENCyclopedia Of DNA Elements) Project. Science 306: 636-640.

Fairbrother WG, Yeh R-F, Sharp PA, Burge CB. 2002. Predictive identification of exonic splicing enhancers in human genes. Science 297: 1007-1013.

Goff LA, Groff AF, Sauvageau M, Trayes-Gibson Z, Sanchez-Gomez DB, Morse M, Martin RD, Elcavage LE, Liapis SC, Gonzalez-Celeiro M, et al. 2015. Spatiotemporal expression and transcriptional perturbations by long noncoding RNAs in the mouse brain. Proc Natl Acad Sci 112: 6855-6862.

Grant CE, Bailey TL, Noble WS. 2011. FIMO: scanning for occurrences of a given motif. Bioinformatics 27: 1017-1018.

Guttman M, Amit I, Garber M, French C, Lin MF, Feldser D, Huarte M, Zuk O, Carey BW, Cassady JP, et al. 2009. Chromatin signature reveals over a thousand highly conserved large non-coding RNAs in mammals. Nature 458: $223-227$.

Hacisuleyman E, Goff LA, Trapnell C, Williams A, Henao-Mejia J, Sun L, McClanahan P, Hendrickson DG, Sauvageau M, Kelley DR, et al. 2014 Topological organization of multichromosomal regions by the long intergenic noncoding RNA Firre. Nat Struct Mol Biol 21: 198-206.

Hacisuleyman E, Shukla CJ, Weiner CL, Rinn JL. 2016. Function and evolution of local repeats in the Firre locus. Nat Commun 7: 11021.

Haerty W, Ponting CP. 2015. Unexpected selection to retain high GC content and splicing enhancers within exons of multiexonic lncRNA loci. RNA 21: 333-346.

Harrow J, Frankish A, Gonzalez JM, Tapanari E, Diekhans M, Kokocinski F, Aken BL, Barrell D, Zadissa A, Searle S, et al. 2012. GENCODE: the reference human genome annotation for The ENCODE Project. Genome Res 22: $1760-1774$

Hezroni H, Koppstein D, Schwartz MG, Avrutin A, Bartel DP, Ulitsky I. 2015. Principles of long noncoding RNA evolution derived from direct comparison of transcriptomes in 17 species. Cell Rep 11: 1110-1122.

Ho DE, Imai K, King G, Stuart EA. 2011. MatchIt: nonparametric preprocessing for parametric causal inference. J Stat Softw 42: 1-28.

Kelley DR, Hendrickson DG, Tenen D, Rinn JL. 2014. Transposable elements modulate human RNA abundance and splicing via specific RNA-protein interactions. Genome Biol 15: 537.

Kim D, Pertea G, Trapnell C, Pimentel H, Kelley R, Salzberg SL. 2013. TopHat2: accurate alignment of transcriptomes in the presence of insertions, deletions and gene fusions. Genome Biol 14: R36.

Kishore S, Jaskiewicz L, Burger L, Hausser J, Khorshid M, Zavolan M. 2011. quantitative analysis of CLIP methods for identifying binding sites of RNA-binding proteins. Nat Methods 8: 559-564.

Kowalczyk MS, Higgs DR, Gingeras TR. 2012. Molecular biology: RNA discrimination. Nature 482: 310-311.

Kutter C, Watt S, Stefflova K, Wilson MD, Goncalves A, Ponting CP, Odom DT, Marques AC. 2012. Rapid turnover of long noncoding RNAs and the evolution of gene expression. PLoS Genet 8: e1002841.

Latos PA, Pauler FM, Koerner MV, Şenergin HB, Hudson QJ, Stocsits RR, Allhoff W, Stricker SH, Klement RM, Warczok KE, et al. 2012. Airn transcriptional overlap, but not its lncRNA products, induces imprinted Igf2r silencing. Science 338: 1469-1472.

Liao J, He Q, Li M, Chen Y, Liu Y, Wang J. 2016. LncRNA MIAT: myocardial infarction associated and more. Gene 578: 158-161.

Lin MF, Jungreis I, Kellis M. 2011. PhyloCSF: a comparative genomics method to distinguish protein coding and non-coding regions. Bioinformatics 27: i275-i282.

Marçais G, Kingsford C. 2011. A fast, lock-free approach for efficient parallel counting of occurrences of $k$-mers. Bioinformatics 27: 764-770.

Marquardt S, Raitskin O, Wu Z, Liu F, Sun Q, Dean C. 2014. Functional consequences of splicing of the antisense transcript COOLAIR on FLC tran scription. Mol Cell 54: 156-165.

Mathelier A, Fornes O, Arenillas DJ, Chen C, Denay G, Lee J, Shi W, Shyr C, Tan G, Worsley-Hunt R, et al. 2016. JASPAR 2016: a major expansion and update of the open-access database of transcription factor binding profiles. Nucleic Acids Res 44: D110-D115. 
Melé M, Rinn JL. 2016. "Cat's cradling" the 3D genome by the act of lncRNA transcription. Mol Cell 62: 657-664.

Melé M, Ferreira PG, Reverter F, DeLuca DS, Monlong J, Sammeth M, Young TR, Goldmann JM, Pervouchine DD, Sullivan TJ, et al. 2015. The human transcriptome across tissues and individuals. Science 348: 660-665.

Mercer TR, Clark MB, Andersen SB, Brunck ME, Haerty W, Crawford J, Taft RJ, Nielsen LK, Dinger ME, Mattick JS. 2015. Genome-wide discovery of human splicing branchpoints. Genome Res 25: 290-303.

Necsulea A, Soumillon M, Warnefors M, Liechti A, Daish T, Zeller U, Baker JC, Grützner F, Kaessmann H. 2014. The evolution of lncRNA repertoires and expression patterns in tetrapods. Nature 505: 635-640.

Pohl A, Beato M. 2014. bwtool: a tool for bigWig files. Bioinformatics 30: $1618-1619$.

Pollard KS, Hubisz MJ, Rosenbloom KR, Siepel A. 2010. Detection of nonneutral substitution rates on mammalian phylogenies. Genome Res 20: $110-121$.

Ponjavic J, Ponting CP, Lunter G. 2007. Functionality or transcriptional noise? Evidence for selection within long noncoding RNAs. Genome Res 17: 556-565.

Quinlan AR, Hall IM. 2010. BEDTools: a flexible suite of utilities for comparing genomic features. Bioinformatics 26: $841-842$.

Quinn JJ, Chang HY. 2015. Unique features of long non-coding RNA biogenesis and function. Nat Rev Genet 17: 47-62.

R Core Team. 2014. R: a language and environment for statistical computing. $\mathrm{R}$ Foundation for Statistical Computing, Vienna, Austria. http://www.Rproject.org/.

Rosenberg AB, Patwardhan RP, Shendure J, Seelig G. 2015. Learning the sequence determinants of alternative splicing from millions of random sequences. Cell 163: 698-711.

Schüler A, Ghanbarian AT, Hurst LD. 2014. Purifying selection on splice-related motifs, not expression level nor RNA folding, explains nearly all constraint on human lincRNAs. Mol Biol Evol 31: 3164-3183.
Seidl CIM, Stricker SH, Barlow DP. 2006. The imprinted Air ncRNA is an atypical RNAPII transcript that evades splicing and escapes nuclear export. EMBO J 25: 3565-3575.

Shao C, Yang B, Wu T, Huang J, Tang P, Zhou Y, Zhou J, Qiu J, Jiang L, Li H, et al. 2014. Mechanisms for U2AF to define $3^{\prime}$ splice sites and regulate alternative splicing in the human genome. Nat Struct Mol Biol 21: 997-1005.

Tilgner H, Knowles DG, Johnson R, Davis CA, Chakrabortty S, Djebali S, Curado J, Snyder M, Gingeras TR, Guigo R. 2012. Deep sequencing of subcellular RNA fractions shows splicing to be predominantly co-transcriptional in the human genome but inefficient for IncRNAs. Genome Res 22: 1616-1625.

Trapnell C, Pachter L, Salzberg SL. 2009. TopHat: discovering splice junctions with RNA-Seq. Bioinformatics 25: $1105-1111$.

Trapnell C, Roberts A, Goff L, Pertea G, Kim D, Kelley DR, Pimentel H, Salzberg SL, Rinn JL, Pachter L. 2012. Differential gene and transcript expression analysis of RNA-seq experiments with TopHat and Cufflinks. Nat Protoc 7: 562-578.

Ulitsky I, Bartel DP. 2013. lincRNAs: genomics, evolution, and mechanisms. Cell 154: $26-46$.

Wang L, Park HJ, Dasari S, Wang S, Kocher J-P, Li W. 2013. CPAT: CodingPotential Assessment Tool using an alignment-free logistic regression model. Nucleic Acids Res 41: e74.

Zarnack K, König J, Tajnik M, Martincorena I, Eustermann S, Stévant I, Reyes A, Anders S, Luscombe NM, Ule J. 2013. Direct competition between hnRNP C and U2AF65 protects the transcriptome from the exonization of Alu elements. Cell 152: 453-466.

Received April 8, 2016; accepted in revised form November 9, 2016. 


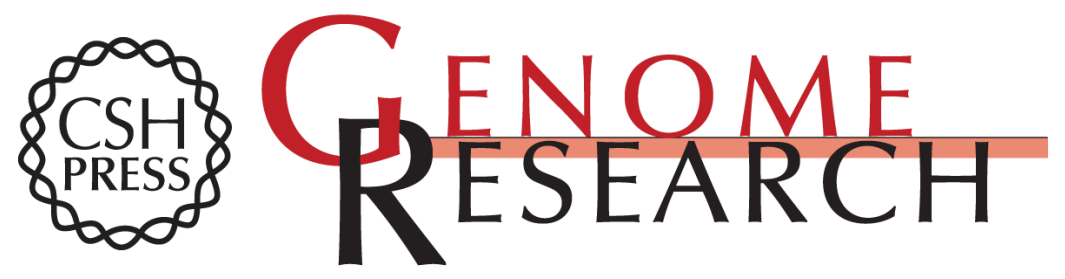

\section{Chromatin environment, transcriptional regulation, and splicing distinguish lincRNAs and mRNAs}

Marta Melé, Kaia Mattioli, William Mallard, et al.

Genome Res. 2017 27: 27-37 originally published online December 7, 2016

Access the most recent version at doi:10.1101/gr.214205.116

Supplemental Material

References

Open Access

Creative Commons

License

Email Alerting Service
http://genome.cshlp.org/content/suppl/2016/12/07/gr.214205.116.DC1

This article cites 52 articles, 16 of which can be accessed free at: http://genome.cshlp.org/content/27/1/27.full.html\#ref-list-1

Freely available online through the Genome Research Open Access option.

This article, published in Genome Research, is available under a Creative Commons License (Attribution-NonCommercial 4.0 International), as described at http://creativecommons.org/licenses/by-nc/4.0/.

Receive free email alerts when new articles cite this article - sign up in the box at the top right corner of the article or click here.

\section{Affordable, Accurate Sequencing.}

